\title{
Towards observational MHD. Advances in spectropolarimetry and the prospects for the E-ELT
}

\author{
Klaus G. Strassmeier \\ Astrophysical Institute Potsdam, An der Sternwarte 16, D-14482 Potsdam, Germany \\ email: kstrassmeier@aip.de
}

\begin{abstract}
Polarization and wavelength are the bits of information attached to every photon that reveal the most about its formation and subsequent history. The E-ELT will, for the foreseeable future, be the most powerful optical light-collecting machine ever built. The strength of its combination, spectropolarimetry with the E-ELT, is the anchorage in physics of astronomical observations. I present a strawman design of a spectropolarimeter for its intermediate focus.
\end{abstract}

Keywords. Magnetic fields - stars: magnetic fields - instrumentation: polarimeters - techniques: polarimetric - techniques: spectroscopic

\section{Size matters}

Per wavelength resolution element, polarization detectable at the $0.1 \%$ level translates into a few million photons. For many classes of objects, even 8-m telescopes quickly run out of power when confronted with such a task. Therefore, as soon as the scientific objectives under consideration reach beyond the mere detection of an object, there is no stronger justification for increased telescope diameters than from spectropolarimetry. In fact, since modern detectors will reach negligible read noise levels, equipment that can measure polarization to better than $0.1 \%$ can also detect very dim sources. In polarimetry, the E-ELT will not do 8-m class science with shorter exposure times. Many of the science cases discussed below $\dagger$ will only be enabled by the E-ELT (see also Baade et al. 2006). This is further compounded by the variability of many types of sources which, in the environment of compact objects, tends to be rapid.

In order to explain complex spectroscopic and photometric observations of accretion processes, collimated jets, stellar rotational braking, coronal heating, non-spherical geometries, and many others, magnetic fields are frequently invoked. But only spectropolarimetry can measure and map magnetic fields and so substitute facts for speculation $\neq$. Figure 1 is a state-of-the-art spectropolarimetric observation of a 11.2-mag star with a $3.6 \mathrm{~m}$ telescope. The polarimetric signal of this star is ten times smaller than the noise, yet it can be reconstructed from time series of such spectra (e.g. Carroll et al. 2011) and used to infer the stellar surface magnetic field. Magnetism is one of the four fundamental forces in nature. Understanding the Universe is impossible without understanding cosmic magnetism, which plays an important role in the formation of celestial bodies and their evolution even during the early stages of the Universe (e.g. Gaenslera et al. 2004) as well as for our own existence on this planet.

$\dagger$ See the full community proposal to ESO at www.eso.org/sci/facilities/eelt/science/doc/

$\ddagger$ As an often-heard joke puts it: "To understand the Universe, we examine galaxies and stars for radiation, small- and large-scale motions, temperatures, chemical composition, and much more. Anything we can't explain after that, we attribute to magnetic fields." 


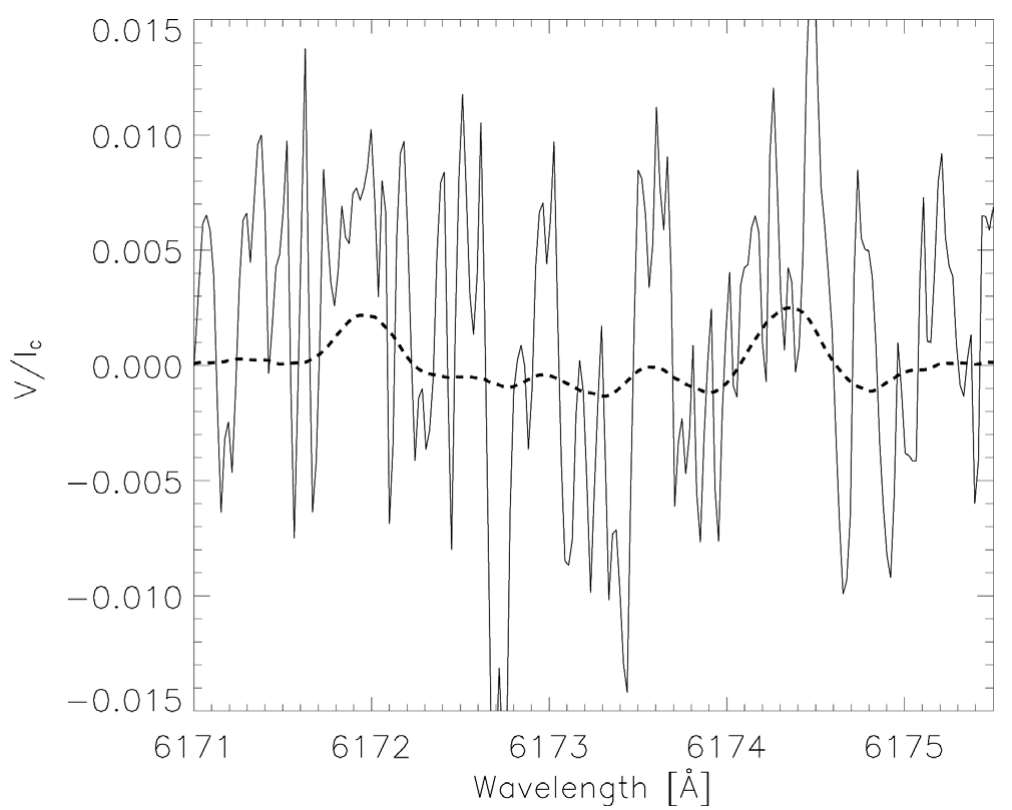

Figure 1. A state-of-the-art spectropolarimetric observation with a $3.6 \mathrm{~m}$ telescope in Stokes V (full line) compared with the signal level that one would expect from a solar-like surface magnetic field (dashed line). Only a small wavelength section around the Fe I-6173 line is shown. The star is V410 Tauri, a weak-lined 11.2-mag T Tauri star, observed with CFHT and the EsPADONS spectropolarimeter at $R=60,000$ and an integration time of $600 \mathrm{~s}$. The magnetic-field signal is approximately ten times smaller than the observational noise.

\section{Some selected science cases}

\subsection{The Vegetation Red Edge and other terrestrial-life markers}

Vegetation has a fivefold higher reflectivity in the NIR than in the optical domain. The steep drop around $700 \mathrm{~nm}$ is called the Vegetation Red Edge (VRE). It has been detected in Earthshine observations off the face of the Moon (e.g. Arnold et al. 2002, Hamdani et al. 2006) and could be a prime target for E-ELT broad-band observations in integrated light. However, the spectral region of the VRE is contaminated by unknown amounts of $\mathrm{O}_{3}$ absorption (plus $\mathrm{O}_{2}$ bands). A possible way out is to observe in polarized light and use the known albedo-polarization relation for planetary surfaces (e.g. Stam 2008). Polarization degrees of up to $20 \%$ are expected from planets in short-period orbits.

The development of terrestrial life may have been helped through seeding by comets. Many molecules are biologically relevant in only one of their two chemically equivalent chiral forms. The origin of this peculiarity is not known. For instance, a chiral imbalance may have arisen from irradiation by circularly polarized starlight, in which case the chirality of terrestrial life would possibly be a mere coincidence. A recently analyzed meteorite suggests that chirality was, in fact, a property of very early solar-system material (Pizzarello et al. 2008). For the more pertinent gaseous matter, circular spectropolarimetry is the analysis method of choice and needs to be applied to cometary tails, the interstellar medium, and even exoplanets. Circular polarization was not detected in the spectrum of the Earthshine yet, which could have been interpreted as a signature of biomatter (chlorophyll in particular) on the Earth's surface (Sterzik \& Bagnulo 2009; see also Boehnhardt et al. 2009). 


\subsection{Protostars: the link to the star-formation process}

Magnetic fields are important ingredients of the star-formation process (McKee \& Ostriker 2007). Models of magnetically driven accretion and outflows successfully reproduce many observational properties of low-mass pre-main sequence stars. Indirect observational evidence for the presence of magnetic fields in these stars manifests itself in strong X-ray, FUV, and UV emission (e.g., Feigelson \& Montmerle 1999).

The first detections of magnetic fields in protostars of class I and II were obtained using NIR spectrographs and revealed kG fields (e.g., Johns-Krull et al. 2009). A VLT surface map in Stokes I of a young star in the Lupus star-forming region even resolved the regions of impacting circumstellar matter and predicted a $3 \mathrm{kG}$ polar field (Strassmeier et al. 2005). The accreted matter is presumably funneled along magnetic field lines but lack of spectropolarimetry prevented the full empirical verification of this model. The first magnetic-field maps of $\mathrm{T}$ Tauri stars show some systems that have complex fields while some have much simpler dipolar/octupolar fields (Donati et al. 2008). Accretion models based on these maps demonstrate the strong dependence of accretion efficiency on both the size and geometry of the star's magnetic field. Fields have also been detected in half a dozen Herbig Ae/Be stars (Hubrig et al. 2009). The magnetic field strength and the X-ray emission of Herbig Ae/Be stars show hints for a decline with age in the range of $\approx 2-14$ Myr supporting a dynamo mechanism that decays with age.

Very few systems can be studied even with $8 \mathrm{~m}$-class telescopes; high-resolution spectropolarimetry at NIR and MIR wavelengths would enable a large step forward in the understanding of star formation. Complex theories have been developed based on indirect indicators of magnetospheric accretion; high-resolution spectropolarimetry in all Stokes parameters is essential in testing them.

\subsection{Interstellar magnetic fields}

Optical and NIR observations can separate dust and magnetic effects and may also be applicable to high-resolution data of the $\mathrm{L} \alpha$ forest in distant quasars. But their potential scope was recently strongly extended by the so-called Atomic Magnetic Realignment effect (Yan \& Lazarian 2008, and references therein). In its basic form, it has higher sensitivity to weak magnetic fields than the Zeeman effect. Its application to emission lines permits the exploration of relatively high-temperature regions. But it mandates extremely large telescope apertures.

Beyond 2-3 kpc from the Sun, the magnetic field of our Galaxy is not well explored (see Feinstein et al. 2008). Magnetic fields in the disk-halo interface regions are complex and only few observations are available. The E-ELT can contribute to many of these questions and generate synergy effects with upcoming radio telescopes, which will dominate in the area of extended sources. Note that optical/NIR measurements can provide the origin of the polarization direction while, in the radio domain, the Faraday effect induces a rotation of the polarization direction. Thus, covering both the radio and the optical/NIR range for such measurements is of high value (see Beck 2010).

\subsection{Type Ia supernovae}

Virtually every supernova that has been properly observed has displayed significant polarization, and hence some significant degree of asymmetry. This has provided new challenges to theory and has shaped the conceptual development of the field.

A particularly stunning reminder of polarimetric diagnostics unparalleled by more limited conventional observing techniques proved to be the observations by Wang et al. (2006) of SN 2004dt. Ca II 3968, Si II 3859/6355, Mg II 4481, and O I 7774 all had about the same velocity profile. Therefore, on a purely spectroscopic basis, one would have 
concluded that all these species have about the same radial distribution in the ejecta. Only polarimetry revealed this as a mistake because, contrary to the other species named, O I 7774 was virtually unpolarized. This is an important finding since of the four elements only oxygen is expected to have been present in major quantities before the explosion. Significant constraints on explosion models arise, which require ELT follow-up, especially with a view towards different metallicities and likely ages inferred from the timing of star bursts.

\section{A strawman design for the E-ELT}

\subsection{Constraints}

The current concept is to provide a simultaneous polarimetric fibre feed to an optical spectrograph like CODEX, or similar, and a NIR spectrograph like SIMPLE, or similar. The applicable wavelength range will be set on the short wavelength side by the fiber throughput as it is rather poor for wavelengths shorter than $380 \mathrm{~nm}$. On the long wavelength side it is set by our intention not to cool the polarimeter optics and thus stay shorter than the thermal IR, say, $1.8 \mu \mathrm{m}$. Currently, the polarimeter exit is laid out to feed both an optical as well as a NIR spectrograph simultaneously although the option to either feed an optical or a NIR instrument alone is retained. A further option is to consider a dedicated, fibre-fed and bench mounted spectrograph of moderately high resolution and fixed format with three arms (Blue, Visual-Red, NIR), somewhat similar to X-Shooter on the VLT but bench mounted.

Such a polarimeter is not foreseen to be operated with laser guide stars, only with natural guide stars. The deformable M4 mirror is only needed in a stiff position. Note that no image derotation is required because all polarimetric targets are observed on the optical axis. However, the polarimeter concept must enable that the acquisition, guiding, and wave-front sensing for M1 is still done through the guide probes in a ring-like FOV with diameter 5-10 arcmin in one of the two Nasmyth foci (which one is tbd). We proceed with a science FOV for the polarimeter of 3 arcsec and try to fit the instrument diameter, its length, and the distance to the focus into the free inner FOV. This comes down to designing a compact collimator that matches these dimensions.

\subsection{Collimator}

The need for a collimator comes from the requirement that the incidence angles into the (plane) polarimetric optical components must be the same across the FOV and remain perpendicular to their respective optical surfaces. Our current design foresees a $15 \mathrm{~mm}$ diameter for this parallel beam. The larger the beam the more problematic is the production of homogeneous crystals with birefringent behavior. The smaller the beam the higher are the requirements on the optical surface quality and homogeneity of the birefringent material. Our overall design is an inverted Schwarzschild system (a microscope in principle) solely based on reflections (Fig. 2). It has the big advantage that cross talk due to birefringency introduced by thermal and mechanical stresses during transmission does not exist. Another advantage is that it is free of chromatic problems, and thus useable for optical and NIR spectrographs at the same time. The disadvantage is that the collimator entrance angles become very large, up to 10 degrees in the present f/4.15 case, and the available FOV very small ( 3 arc sec in our case).

\subsection{Polarimetric components}

The first component of the polarimeter is the linear polarizer and beam splitter, in our case we propose a non-standard Foster prism following the PEPSI design (Strassmeier 


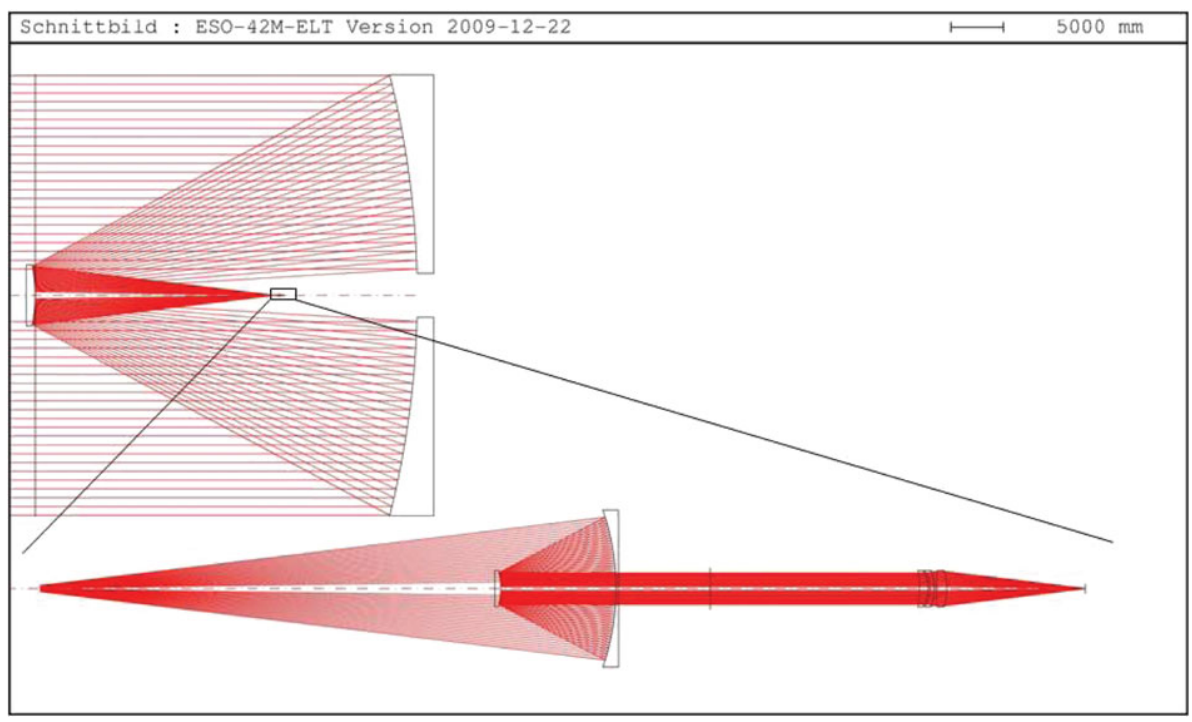

Figure 2. Optical design of a collimator for the intermediate f/4.15 focus of the E-ELT. The top drawing shows the telescope together with the collimator (in the box as indicated). The bottom drawing is a blow-up of the collimator design alone. The polarimetric components are located in the parallel beam before the camera (only one beam and one camera is shown).

et al. 2008). Currently, the calcite for such a prism is principally - but not easily available and may require additional $\mathrm{R} \& \mathrm{D}$ with the optical industry (e.g. Spanó et al. 2006). The entire unit consists of three prisms, two are made of calcite, one is N-Bk7. The latter is needed to make the output ordinary (o)- and extra-ordinary (eo)-beams parallel to each other. This enables a compact and slim mechanical design in order to minimize diffraction of the telescope beam. In linear polarizing mode, the Foster unit will be rotated and thus must be turnable with respect to the sky by $270^{\circ}$.

The second component of the polarimeter is the elliptical retarder in front of the Foster subunit. It must be additionally rotateable with respect to the Foster unit. We envision to use a similar retarder type that we implemented in the PEPSI spectropolarimeter for the LBT as well as for the calibration unit for the GREGOR 1.5m solar telescope on Tenerife. These retarders are based on plastic sheets (polymethylmetacrylat) and we envision a single, elliptical retarder for the entire wavelength range from $380-1800 \mathrm{~nm}$. This will require some $\mathrm{R} \& \mathrm{D}$ with the optical industry but appears reasonable.

\subsection{Dichroic beam splitters}

For our conceptual design, we decided to proceed with two simultaneous wavelength regions, optical and NIR, i.e. feeding proposed instruments like CODEX and SIMPLE at the same time. The wavelength cut is set at $1000 \mathrm{~nm}$ with wings extending $\pm 30 \mathrm{~nm}$. The dichroic beam splitter would be situated behind the Foster subunit and behind a common ADC for both beams (o and eo) and follow the design for a multi-channel focal reducer by Laux et al. (2009).

\section{Conclusions}

The special and unique sensitivity of polarization to the interaction between light and matter gives polarimetry diagnostic qualities not available with other observing techniques. However, amongst the recent and near-future infrared space missions (Spitzer, 
Herschel, JWST), there is no polarimetric option. In space there is an obvious imperative to keep mechanical systems as simple and trouble-free as possible, and in wavelength regions with useful atmospheric transmission, polarimetry can be achieved more effectively from the ground. Yet, the 2007 proposal for the construction for the US Thirty Meter Telescope ignores polarimetry, except in the context of the search for extra-solar planets. Similarly, the Science Case from 2006 for the Giant Magellan Telescope only mentions supernovae polarization explicitly. However, large-area polarization surveys are foreseen with the LSST (Clemens et al. 2009), or are even ongoing at IRSF (Kandori et al. 2006), and will provide a wealth of polarized targets for the E-ELT.

I conclude that a megastructure like the E-ELT with its $1380 \mathrm{~m}^{2}$ light-collecting area should be in the position to recover the full wavefront information.

\section{Acknowledgements}

I would like to thank Alfio Bonanno and his team for a great meeting. Also thanks to my AIP colleagues Thorsten Carroll, Michi Weber and Thomas Granzer for continuing discussions and Uwe Laux for his efforts in the optical design of the collimator. The EELT science case for a spectropolarimeter was a joint effort of 80 colleagues across Europe with particular input from Dietrich Baade at ESO, whom I thank for this. I acknowledge grant STR645-1 from the Deutsche Forschungsgemeinschaft (DFG) and the support from the Bundeministerium für Bildung \& Forschung (BMBF) through the Verbundforschung grant 05A09IPA.

\section{References}

Arnold, L., Gillet, S., Lardiere, O., Riaud, \& P., Schneider, \& J. 2002, A\& A 392, 231

Baade, D., Wang, L., Hubrig, S., \& Patat, F. 2006, in IAU Symp. 232, CUP, p.248

Beck, R. 2010, in "Astronomy with Megastructures. Joint Science with E-ELT and SKA", arXiv:1008.3806

Boehnhardt, H., Tozzi, G. P. Sterzik, M. et al. 2009, Earth, Moon $E S$ Planets, 105, 95

Carroll, T. A., Strassmeier, K. G., Ilyin, I., \& Rice, J. B. 2011, in IAU Symp. 273, CUP, in press

Clemens, D. P., Pinnick, A., Pavel, M. et al. 2009, BAAS 41, 459

Donati, J.-F. et al. 2008, MNRAS 386, 1234

Feigelson, E. \& Montmerle, T. 1999, ARA\&A 37, 363

Feinstein, C., Vergne, M. M., Martinez, R., \& Orsatti, A. M. 2008, MNRAS 391, 447

Gaenslera, B. M., Beck, R., \& Feretti, L. 2004, New Astron. Reviews 48, 1003

Hamdani, S., Arnold, L., Foellmi, C. et al. 2006, A\&A 460, 617

Hubrig, S., Grady, C., Schöller, M. et al. 2009, in IAU Symp. 259, CUP, p.395

Johns-Krull, C., Greene, T. P., Doppmann, G. W., \& Covey, K. R. 2009, ApJ 700, 1440

Kandori, R., Kusakabe, N., Tamura, M. et al. 2006, SPIE 6269, 159

Laux, U., Klose, S., \& Greiner, J. 2009, poster at the fall meeting of the AG 2009, Potsdam

McKee, C. F. \& Ostriker, E. C. 2007, ARA\&A 45, 565

Pizzarello, S. et al. 2008, PNAS 105, 3010

Spanó, P., Zerbi, F. M., Norrie, C. J. et al. 2006, AN 327, 649

Stam, D. M. 2008, A\&A 482, 989

Sterzik, M. F. \& Bagnulo, S. 2009, in Bioastronomy 2007, ASPC 420, p.371

Strassmeier, K. G., Rice, J. B., Ritter, A. et al. 2005, A\&SA 440, 1105

Strassmeier, K. G., Woche, M., Ilyin, I. et al. 2008, SPIE 7014, 21

Wang, L., Baade, D., Höflich, P. et al. 2006, ApJ 653, 490

Yan, H. \& Lazarian, A. 2008, ApJ 677, 1401 